\title{
Strong covering without squares
}

\author{
by
}

Saharon Shelah (Jerusalem and New Brunswick, NJ)

\begin{abstract}
Let $W$ be an inner model of ZFC. Let $\kappa$ be a cardinal in $V$. We say that $\kappa$-covering holds between $V$ and $W$ iff for all $X \in V$ with $X \subseteq$ ON and $V \models|X|<\kappa$, there exists $Y \in W$ such that $X \subseteq Y \subseteq O N$ and $V \models|Y|<\kappa$. Strong $\kappa$-covering holds between $V$ and $W$ iff for every structure $\mathcal{M} \in V$ for some countable first-order language whose underlying set is some ordinal $\lambda$, and every $X \in V$ with $X \subseteq \lambda$ and $V \models|X|<\kappa$, there is $Y \in W$ such that $X \subseteq Y \prec M$ and $V \models|Y|<\kappa$.

We prove that if $\kappa$ is $V$-regular, $\kappa_{V}^{+}=\kappa_{W}^{+}$, and we have both $\kappa$-covering and $\kappa^{+}-$ covering between $W$ and $V$, then strong $\kappa$-covering holds. Next we show that we can drop the assumption of $\kappa^{+}$-covering at the expense of assuming some more absoluteness of cardinals and cofinalities between $W$ and $V$, and that we can drop the assumption that $\kappa_{W}^{+}=\kappa_{V}^{+}$and weaken the $\kappa^{+}$-covering assumption at the expense of assuming some structural facts about $W$ (the existence of certain square sequences).
\end{abstract}

1. Introduction. The study of "covering lemmas" started with Jensen [DeJe] who proved in 1974-5 that in the absence of $0^{\sharp}$ there is a certain degree of resemblance between $V$ and $L$. More precisely, if $0^{\sharp}$ does not exist then for every set of ordinals $X$ there exists a set of ordinals $Y \in L$ such that $X \subseteq Y$ and $V \models|Y|=\max \left\{|X|, \aleph_{1}\right\}$. There is no hope of covering countable sets by countable ones in general, because doing Namba forcing over $L$ will change the cofinality of $\aleph_{2}^{L}$ to $\omega$ while preserving $\aleph_{1}$.

This form of covering has strong implications for the structure of $V$. For example Jensen's theorem implies that in the absence of $0^{\sharp}$ the Singular Cardinals Hypothesis holds, and that there is a special $\kappa^{+}$-Aronszajn tree for every singular $\kappa$. So we can conclude that the negations of these statements have substantial consistency strength.

One subsequent line of development has involved proving covering lemmas over larger and larger "core models", on the assumption of the non-

2000 Mathematics Subject Classification: 03E04, 03E05, 03E45.

Key words and phrases: set theory, covering, strong covering lemma, pcf theory.

The research was partially supported by "Israel Science Foundation", founded by the Israel Academy of Sciences and Humanities. Publication 580. 
existence of stronger and stronger large cardinals. Inevitably these covering lemmas have much more complex statements than Jensen's original theorem, the reason being that once the core model contains even one measurable cardinal we can start to do Prikry forcing.

This line of research has provided much information about the consistency strengths of combinatorial hypotheses. For example work of Gitik and Mitchell has determined the exact strength of the failure of the Singular Cardinals Hypothesis, while work of Schimmerling, Mitchell and Steel has provided a very strong lower bound (a Woodin cardinal) for the strength of " $\kappa$ is singular and there is no special $\kappa^{+}$-Aronszajn tree".

Another line of development involved getting more information about the nature of the covering set $Y$. For example given an ordinal $\lambda$ and some first-order structure $\mathcal{M} \in V$ with underlying set $\lambda$, we can take a set $X \subseteq \lambda$ and ask whether it can be covered by some $Y \in W$ with $|Y| \leq \max \left\{|X|, \aleph_{1}\right\}$ and $Y \prec \mathcal{M}$. This kind of phenomenon is called strong covering (see Definition 1.1; for history see [Sh:g, Ch. VII, pp. 276-277]).

One approach to proving strong covering theorems is to go back to Jensen's proof and to prove directly that there exists an appropriate $Y \in L$. This approach was taken by Carlson [Ca]. Another approach (due to the author) is more axiomatic; given two transitive class models $W \subseteq V$ of ZFC where $W$ is sufficiently $L$-like and for every $X \in V$ there is $Y \supseteq X$ with $|Y|=\max \left\{|X|, \aleph_{1}\right\}$, it is proved in [Sh:b, XIII] that a certain form of strong covering holds between $V$ and $W$.

The work in this paper continues that in [Sh:b, XIII] and [Sh410] (note that a slightly improved version of [Sh:b, XIII] has appeared as [Sh:g, VII]). The idea here is to eliminate as far as possible the structural assumptions on $W$. We start by outlining the structure of the paper.

1.1. Definition. Let $W$ be an inner model of ZFC. Let $\kappa$ be an uncountable cardinal in $V$.

(1) $\kappa$-covering holds between $V$ and $W$ iff for all $X \in V$ with $X \subseteq$ ON and $V \models|X|<\kappa$, there exists $Y \in W$ such that $X \subseteq Y \subseteq$ ON and $V \models|Y|<\kappa$.

(2) Strong $\kappa$-covering holds between $V$ and $W$ iff for every structure $\mathcal{M} \in V$ for some countable first-order language whose underlying set is some ordinal $\lambda$, and every $X \in V$ with $X \subseteq \lambda$ and $V \models|X|<\kappa$, there is $Y \in W$ such that $X \subseteq Y \prec M$ and $V \models|Y|<\kappa$.

In the first section it is proved that if $\kappa$ is $V$-regular, $\kappa_{V}^{+}=\kappa_{W}^{+}$, and we have both $\kappa$-covering and $\kappa^{+}$-covering between $W$ and $V$, then strong $\kappa$-covering holds. In fact something rather stronger is proved. The assumption that $\kappa$-covering holds is reasonable enough, but we can hope to weaken the other assumptions. 
In the rest of the paper we prove a series of facts about covering culminating in two main results; one result says that we can drop the assumption of $\kappa^{+}$-covering at the expense of assuming some more absoluteness of cardinals and cofinalities between $W$ and $V$, and the other says that we can drop the assumption that $\kappa_{W}^{+}=\kappa_{V}^{+}$and weaken the $\kappa^{+}$-covering assumption at the expense of assuming some structural facts about $W$ (the existence of certain square sequences). Both these results are contained in Theorem 7.1.

The paper was written up by Uri Abraham and James Cummings, and I am grateful for their excellent work. I am also grateful to Moti Gitik for asking me about the possibility of a theorem like Theorem 7.1 after reading [Sh420].

The material in this paper represents part of some lectures given by the author in Jerusalem in the period May-August 1995. The rest of those lectures will appear in [Sh598] and so we have retained here to some extent the notation and terminology used in the lectures. Generally see [Sh:E12].

In particular the Jerusalem lectures introduced names for some of the important hypotheses. For the record, here is a complete list of those names. In the body of the paper we will recall these definitions as and when we need them. $W$ will always be some inner model of ZFC.
(A) ${ }_{\mu, \lambda}$
(B) ${ }_{\mu, \lambda}$
(B) ${ }_{\mu}$
$(\mathrm{B})_{\mu, \lambda}^{-}$
$(\mathrm{B})_{\mu, \lambda}^{*}$
(C) ${ }_{\mu, \lambda}$
(D) ${ }_{\mu, \lambda}$

$(\mathrm{E})_{\mathcal{P}}$

$(\mathrm{F})_{\mathbb{D}, \lambda, \mu}$

$(\mathrm{G})_{\mathbb{D}, \mathcal{P}, \mu}$

$(\mathrm{H})_{\mathbb{D}, \mu}$

$$
\aleph_{0}<\mu=\operatorname{cf}_{V}(\mu)<\lambda \in \mathrm{CARD}^{W} \text {. }
$$

$[\lambda]_{W}^{<\mu}$ is cofinal in $[\lambda]^{<\mu}$.

(B) ${ }_{\mu, \lambda}$ holds for every $\lambda$.

For all $\delta \leq \lambda, \operatorname{cf}^{W}(\delta)<\mu$ iff $\operatorname{cf}(\delta)<\mu$.

For all $A \in[\lambda]^{<\mu}$ there is $B \in[\lambda]_{W}^{<\mu_{V}^{+}}$such that $A \subseteq B$.

For all $\mathfrak{a} \in W$, if $\mathfrak{a} \subseteq \mathrm{REG}^{W} \cap \lambda \backslash \mu$ and $W \models$ " $|\mathfrak{a}|<\mu$ " then $\max \operatorname{pcf}_{W}(\mathfrak{a}) \leq \lambda$.

In $W$ there is a map $\theta \mapsto \vec{C}^{\theta}$ such that for each $\theta \in \mathrm{REG}^{W} \cap$ $(\mu, \lambda], \vec{C}^{\theta}=\left\langle C_{\alpha}^{\theta}: \alpha<\theta, \operatorname{cf}_{W}(\alpha)<\mu\right\rangle$ is a sequence such that $C_{\alpha}^{\theta}$ is club in $\alpha$, o.t. $\left(C_{\alpha}^{\theta}\right)<\mu$, and $\beta \in \lim \left(C_{\alpha}^{\theta}\right) \Rightarrow C_{\alpha}^{\theta} \cap \beta=C_{\beta}^{\theta}$. (A square in $\theta$ on points of cofinality less than $\mu$.)

$W \models " \mathcal{P} \subseteq[\lambda]^{<\mu},|\mathcal{P}|=\lambda "$.

For every $x \in[\lambda]^{\mu}$ there exists $\left\langle a_{i}: i<\mu\right\rangle$ with $a_{i} \in[\lambda]^{<\mu}$ increasing and continuous such that $\left|x \cap \bigcup_{i} a_{i}\right|=\mu,\left\{i<\mu \mid a_{i} \in W\right\}$ $\in \mathbb{D}$. Here $\mathbb{D}$ is a normal filter on $\mu$. If $\mathbb{D}$ is the club filter on $\mu$, we may omit it. (See more after 3.1.)

For every increasing sequence $\left\langle b_{i}: i<\mu\right\rangle$ with $b_{i} \in \mathcal{P}$ for all $i$, $\left\{i<\mu \mid \bigcup_{j<i} b_{j} \in W\right\} \in \mathbb{D}$.

For all $\alpha \in\left(\mu, \mu^{+}\right)$, if $\alpha=\bigcup_{i<\mu} a_{i}, a_{i} \in[\alpha]^{<\mu}$, with $\vec{a}$ increasing and continuous then $\left\{i<\mu \mid a_{i} \in W\right\} \in \mathbb{D}$. Here $\mathbb{D}$ is a normal filter on $\mu$. If $\mathbb{D}$ is the club filter on $\mu$, we omit it. 
(I) $)_{\mu, \lambda} \quad$ If $W \models " \mathfrak{a} \subseteq(\lambda+1) \backslash \mu^{+},|\mathfrak{a}|<\mu$ " then $\max \operatorname{pcf}_{W}(\mathfrak{a}) \leq \lambda$.

$(\mathrm{J})_{\mu, \lambda} \quad$ Given a sequence $\left\langle\mathfrak{a}_{\alpha}: \alpha<\mu\right\rangle$ such that $\mathfrak{a}_{\alpha} \in W, \mathfrak{a}_{\alpha} \subseteq \mathrm{REG}^{W} \cap$ $\lambda \backslash \mu^{+},\left|\mathfrak{a}_{\alpha}\right|<\mu$, along with $f \in \prod\left(\bigcup_{\alpha} \mathfrak{a}_{\alpha}\right)$, there exists $g \in$ $(\mathrm{K})_{\mu, \lambda} \quad W \models \operatorname{cf}\left([\lambda]^{<\mu}, \subseteq\right)=\lambda$.

The logical structure of the paper is given by the following picture, where $\kappa$ is regular uncountable in $V, \mathcal{P} \in W, \mathcal{P} \subseteq[\lambda]^{<\kappa}$.

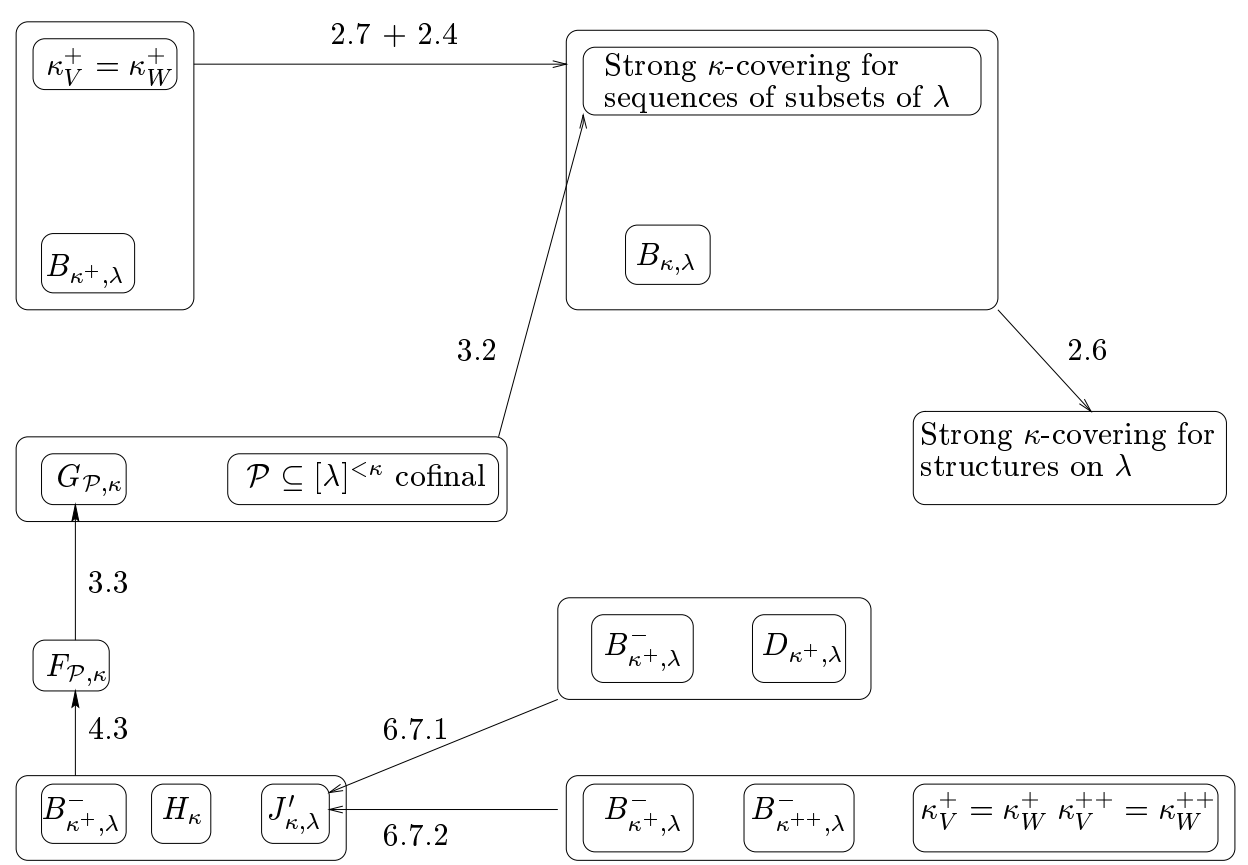

Fig. 1. The structure of the proof

We conclude with a few words about notation. If $\tau$ is some set-theoretic term then " $\tau_{M}$ " will mean "the result of interpreting $\tau$ in the model $M$ ". When we write " $M \models \phi(\tau)$ " we mean that " $\phi$ holds of $\tau_{M}$ in the sense of the model $M$ ". For example " $L \models \operatorname{cf}(\alpha)=\omega_{2}$ " means the same thing as "cf $f_{L}(\alpha)=\left(\omega_{2}\right)_{L}$ ".

2. The painless strong covering theorem. In this section we will give a simple proof of a form of strong covering from rather strong hypotheses. We begin by discussing the well known concept of a "filtration" which will be useful at several points in what follows.

2.1. Definition. Let $\mu$ be a regular uncountable cardinal and let $X$ be a set of cardinality $\mu$. Then a filtration of $X$ is a sequence $\vec{X}=\left\langle X_{\alpha}: \alpha<\mu\right\rangle$ such that: 
(1) $\left|X_{\alpha}\right|<\mu$ for all $\alpha<\mu$.

(2) $\alpha<\beta \Rightarrow X_{\alpha} \subseteq X_{\beta}$.

(3) For limit $\lambda, X_{\lambda}=\bigcup_{\alpha<\lambda} X_{\alpha}$.

(4) $X=\bigcup_{\alpha<\mu} X_{\mu}$.

The key fact about filtrations is that they are in a sense unique.

2.2. LemmA. If $\vec{X}$ and $\vec{X}^{\prime}$ are two filtrations of a set $X$ of cardinality $\mu$, then $A:=\left\{\alpha<\mu \mid X_{\alpha}=X_{\alpha}^{\prime}\right\}$ is closed and unbounded in $\mu$.

Proof. For the closure, let $\lambda$ be a limit point of $A$, and observe that then

$$
X_{\lambda}=\bigcup_{\alpha<\lambda} X_{\alpha}=\bigcup_{\alpha \in A \cap \lambda} X_{\alpha}=\bigcup_{\alpha \in A \cap \lambda} X_{\alpha}^{\prime}=X_{\lambda}^{\prime},
$$

so $\lambda \in A$. For unboundedness, fix $\alpha_{0}<\mu$. Now $X_{\alpha_{0}} \subseteq X=\bigcup_{\alpha<\mu} X_{\alpha}^{\prime}$, $\left|X_{\alpha_{0}}\right|<\mu$ and $\mu$ is regular, so for some $\beta_{0}<\mu$ we have $X_{\alpha_{0}} \subseteq X_{\beta_{0}}^{\prime}$. Arguing in a similar vein we may find $\alpha_{0}<\beta_{0}<\alpha_{1}<\beta_{1}<\ldots$ such that $X_{\alpha_{0}} \subseteq X_{\beta_{0}}^{\prime} \subseteq X_{\alpha_{1}} \subseteq X_{\beta_{1}}^{\prime} \subset \ldots$ If now $\lambda=\sup _{n} \alpha_{n}=\sup _{n} \beta_{n}$, then $X_{\lambda}=\bigcup_{n} X_{\alpha_{n}}=\bigcup_{n} X_{\beta_{n}}^{\prime}=X_{\lambda}^{\prime}$ so that $\lambda \in A$ and we have proved that $A$ is unbounded.

Recall that the hypothesis $(\mathrm{B})_{\mu}$ says that if $X \in V, X \subseteq \mathrm{ON}$ and $V \models|X|<\mu$ then there is $Y \in W, X \subseteq Y \subseteq$ ON with $V \models|Y|<\mu$. This is the hypothesis which we called " $\mu$-covering" in the introduction.

2.3. TheORem. Let $\kappa$ be regular and uncountable in $V$. Suppose that $(\mathrm{B})_{\kappa}$ and $(\mathrm{B})_{\kappa^{+}}$both hold and that $\kappa_{V}^{+}=\kappa_{W}^{+}$. Let $\mathcal{M} \in V$ be a structure for some countable first order language, whose underlying set is an ordinal $\lambda \geq \kappa$. Let $z$ be a set in $[\lambda]^{<\kappa}$. Then there exists an increasing continuous sequence of sets $\left\langle z_{\alpha}: \alpha<\kappa\right\rangle$ such that $z \subseteq z_{0},\left|z_{\alpha}\right|<\kappa, z_{\alpha} \prec \mathcal{M}$, $z_{\alpha} \in W$, and $\alpha \subseteq z_{\alpha}$. In particular, strong $\kappa$-covering holds between $V$ and $W$.

Pr o of. We build an increasing continuous sequence $\left\langle x_{\alpha}: \alpha<\kappa\right\rangle$ of subsets of $\lambda$, where each $x_{\alpha}$ has size less than $\kappa$.

- In case $\alpha=0, x_{0}=z$.

- In case $\alpha=2 \beta+1, x_{\alpha}$ is some set such that $x_{2 \beta} \subsetneq x_{\alpha} \subseteq \lambda, x_{\alpha} \in W$, $\alpha+1 \subseteq x_{\alpha},\left|x_{\alpha}\right|<\kappa$. Such a set exists because we are assuming $(\mathrm{B})_{\kappa}$.

- In case $\alpha=2 \beta+2, x_{\alpha}$ is some set such that $x_{2 \beta+1} \subsetneq x_{\alpha} \subseteq \lambda, x_{\alpha} \prec \mathcal{M}$, and $\left|x_{\alpha}\right|<\kappa$. Such a set exists because $\mathcal{M}$ has a countable set of Skolem functions and $\kappa$ is uncountable.

- In case $\alpha$ is limit, $x_{\alpha}=\bigcup_{\beta<\alpha} x_{\beta}$. Then $\left|x_{\alpha}\right|<\kappa$ because $\kappa$ is regular. Observe that $x_{\alpha} \prec \mathcal{M}$, because $x_{\alpha}$ is the union of the increasing chain of substructures $\left\langle x_{2 \beta+2}: 2 \beta+2<\alpha\right\rangle$. 
Now let $x=\bigcup_{\alpha<\kappa} x_{\alpha}$, so that $|x|=\kappa$. Applying the hypothesis $(\mathrm{B})_{\kappa^{+}}$, we may find $y \subseteq \lambda$ such that $y \in W, x \subseteq y$ and $V \models|y|<\kappa^{+}$. Since we know that $\kappa_{V}^{+}=\kappa_{W}^{+}$, we see that in fact $W \models|y|=\kappa$. Working in $W$, let us fix a filtration $\left\langle y_{\alpha}: \alpha<\kappa\right\rangle$ of $y$.

Now we observe that we have two filtrations of the set $x$, because $x=$ $\bigcup_{\alpha} x_{\alpha}=\bigcup_{\alpha}\left(x \cap y_{\alpha}\right)$. By Lemma 2.2 we can find a club $C$ of limit ordinals in $\kappa$ such that $\alpha \in C \Rightarrow x_{\alpha}=x \cap y_{\alpha}$. Now $x_{\alpha} \subseteq x_{\alpha+1} \subseteq x$ so that for $\alpha \in C$,

$$
x_{\alpha}=x_{\alpha} \cap x_{\alpha+1}=x \cap y_{\alpha} \cap x_{\alpha+1}=y_{\alpha} \cap x_{\alpha+1},
$$

and hence $x_{\alpha} \in W$ since both $x_{\alpha+1}$ and $y_{\alpha}$ are in $W$. If we enumerate $C$ in increasing order as $\left\langle\gamma_{\alpha}: \alpha<\kappa\right\rangle$ and set $z_{\alpha}=x_{\gamma_{\alpha}}$ then $\left\langle z_{\alpha}: \alpha<\kappa\right\rangle$ is continuous and increasing, $z_{\alpha} \prec M$ and $z_{\alpha} \in W$ for all $\alpha<\kappa$.

2.4. Remark. The assumption $(\mathrm{B})_{\kappa}$ in the theorem is redundant, because actually the assumptions $(\mathrm{B})_{\kappa^{+}}$and $\kappa_{V}^{+}=\kappa_{W}^{+}$imply $(\mathrm{B})_{\kappa}$. For if $|X|<\kappa$ we may cover it by $Y \in W$ such that $|Y|_{V}<\kappa^{+}$, but then by the agreement between cardinals we must have $|Y|_{W} \leq \kappa$. Now in $W$ we write $Y=\bigcup_{i<\kappa} Y_{i}$ with $\left|Y_{i}\right|<\kappa$, and since $\kappa$ is regular in $V$ there exists $i$ such that $X \subseteq Y_{i}$.

We can analyse the proof of Theorem 2.3 into two steps, an analysis which gives some motivation for the work of later sections.

2.5. Definition. Let $\kappa>\aleph_{0}$ be regular, and let $W$ be an inner model.

(1) A set $X \in V$ with $|X|=\kappa$ and $X \subseteq W$ is $W$-filtered iff there is a filtration $\vec{X}$ of $X$ such that $X_{\alpha} \in W$ for a closed unbounded set of $\alpha<\kappa$.

(2) Strong $\kappa$-covering for sequences holds between $V$ and $W$ for subsets of $\lambda$ iff whenever $\left\langle a_{i}: i<\kappa\right\rangle$ is an increasing (but not necessarily continuous) sequence of subsets of $\lambda$ with $a_{i} \in W$ and $\left|a_{i}\right|<\kappa$, then $\bigcup_{i} a_{i}$ is $W$-filtered.

(3) Strong $\kappa$-covering for sequences holds between $V$ and $W$ iff for all $\lambda$ strong $\kappa$-covering for sequences holds between $V$ and $W$ for subsets of $\lambda$.

The proof of Theorem 2.3 can be broken into two lemmas.

2.6. Lemma. Let $(\mathrm{B})_{\kappa}$ hold, let $\mathcal{M}$ be a structure for a countable firstorder language with underlying set $\lambda$, and let $z \subseteq \lambda$ with $|z|<\kappa$. Then $z \subseteq \bigcup_{i<\kappa} a_{i}$ where $\left|a_{i}\right|<\kappa, a_{i} \in W$ for $i$ odd, $a_{i} \prec M$ for $i$ even, $a_{i}$ increasing continuous.

2.7. Lemma. If $(\mathrm{B})_{\kappa^{+}}$and $\kappa_{V}^{+}=\kappa_{W}^{+}$then strong $\kappa$-covering for sequences holds.

It follows from the second lemma that for many limit $i$ we have $a_{i} \in W$ and hence we have covered $z$ by a substructure lying in $W$. 
2.8. Remark. Notice that by Lemma 2.2 , if $X$ is $W$-filtered then for any filtration $\vec{X}$ we have $X_{\alpha} \in W$ for a club of $\alpha$. Another equivalent definition of " $W$-filtered" would demand that $X$ has a filtration such that $X_{\alpha} \in W$ for every $\alpha$.

2.9. REMARK.Strong $\kappa$-covering for sequences does not imply $\kappa$-covering. For example if $L[G]$ is the generic extension of $L$ for Namba forcing then strong $\omega_{1}$-covering for sequences holds (by an easy argument using the fact that Namba forcing adds no reals to a model of $\mathrm{CH}$ ) but $\omega_{1}$-covering certainly fails.

3. (F) implies (G). We now begin to show how to weaken the hypotheses of Theorem 2.3 from the last section. Throughout this section $\kappa$ denotes a regular uncountable cardinal (in $V$ ), and $\lambda$ a $W$-cardinal with $\lambda>\kappa$.

The next lemma represents a variation on the main idea in Theorem 2.3. Here we are covering by a set of size $\kappa$ which is $W$-filtered, rather than actually lying in $W$.

3.1. Lemma. Suppose that $b=\bigcup_{i<\kappa} b_{i}$ where $b_{i} \in W,\left|b_{i}\right|<\kappa, b_{i} \subseteq b_{j}$ for $i<j<\kappa$. If $b$ is included in a $W$-filtered set of size $\kappa$, then $b$ itself is $W$-filtered.

Proof. Suppose that $b \subseteq a$ where $a$ is $W$-filtered, as evidenced by an increasing continuous sequence $\left\langle a_{i}: i<\kappa\right\rangle$ such that $a=\bigcup_{i<\kappa} a_{i}, a_{i} \in W$, $\left|a_{i}\right|<\kappa$. Then the sets $a_{i} \cap b$ form one filtration of $b$, while $\left\langle\bigcup_{i<\delta} b_{i}: \delta<\kappa\right\rangle$ is another. By Lemma 2.2,

$$
D=\left\{\delta<\kappa \mid a_{\delta} \cap b=\bigcup_{\alpha<\delta} b_{\alpha}\right\}
$$

contains a club set. We will prove that $a_{\delta} \cap b \in W$ for every $\delta \in D$. Since the sequence of $b_{\alpha}$ 's is increasing, $\bigcup_{\alpha<\delta} b_{\alpha} \subseteq b_{\delta}$. Hence since $a_{\delta} \cap b=\bigcup_{\alpha<\delta} b_{\alpha}$, we have $a_{\delta} \cap b=a_{\delta} \cap b_{\delta}$ as well. Hence, if $\delta \in D$, then $a_{\delta} \cap b \in W$ (since $\left.a_{\delta}, b_{\delta} \in W\right)$.

Recall that $(\mathrm{F})_{\lambda, \kappa}$ denotes the following statement:

$(\mathrm{F})_{\lambda, \kappa} \quad$ For all $x \in[\lambda]^{\kappa}$ there exists $A$ such that $|A|=\kappa, A$ is $W$-filtered and $|x \cap A|=\kappa$.

Let $\mathcal{P}$ be such that $W \models " \mathcal{P} \subseteq[\lambda]^{<\kappa},|\mathcal{P}|=\lambda$ ". In applications of $(\mathrm{F})_{\lambda, \kappa}$ we will replace $\lambda$ with $\mathcal{P}$, and then $(\mathrm{F})_{\lambda, \kappa}$ becomes the following sentence:

$(\mathrm{F})_{\mathcal{P}, \kappa} \quad$ For all $x \in[\mathcal{P}]^{\kappa}$ there exists $A$ such that $|A|=\kappa, A$ is $W$-filtered and $|x \cap A|=\kappa$.

Clearly for $\mathcal{P} \in W$ we have $(\mathrm{F})_{\mathcal{P}, \kappa} \Leftrightarrow(\mathrm{F})_{|\mathcal{P}|, \kappa}$.

Recall also that we defined 
$(\mathrm{G})_{\mathcal{P}, \kappa} \quad$ For every $\subseteq$-increasing sequence $\left\langle b_{\alpha}: \alpha<\kappa\right\rangle$ of sets with $b_{\alpha} \in \mathcal{P}$ for all $\alpha$, the set $\left\{\delta<\kappa \mid \bigcup_{\alpha<\delta} b_{\alpha} \in W\right\}$ contains a club set.

Equivalently, $(\mathrm{G})_{\mathcal{P}, \kappa}$ says that for every $\subseteq$-increasing sequence $\left\langle b_{\alpha}: \alpha\right.$ $\langle\kappa\rangle$ with $b_{\alpha} \in \mathcal{P}$, if $b=\bigcup_{\alpha<\kappa} b_{\alpha}$ then $b$ is $W$-filtered. This is a weakening of the statement of strong $\kappa$-covering for sequences, where we restrict to the class of increasing sequences from $\mathcal{P}$.

3.2. Lemma. If $(\mathcal{P}, \subseteq)$ is cofinal in $\left(\lambda^{<\kappa}, \subseteq\right)$, then $(\mathrm{G})_{\mathcal{P}, \kappa}$ implies the strong $\kappa$-covering for sequences of subsets of $\lambda$.

Proof. Let $\left\langle a_{i}: i<\kappa\right\rangle$ be an increasing sequence of subsets of $\lambda$ such that $a_{i} \in W$ and $\left|a_{i}\right|<\kappa$. It is required to prove that $a=\bigcup_{i<\kappa} a_{i}$ is $W$ filtered. By Lemma 3.1 it suffices to prove that $a$ is included in a $W$-filtered set, and for this we define inductively an increasing sequence $b_{i} \in \mathcal{P}$ for $i<\kappa$ such that $a_{i} \subseteq b_{i}$. Now by $(\mathrm{G})_{\mathcal{P}, \kappa}$ the set $b=\bigcup_{i<\kappa} b_{i}$ is $W$-filtered, and $a \subseteq b$, hence $a$ is $W$-filtered.

\subsection{TheOREM. $(\mathrm{F})_{\mathcal{P}, \kappa}$ implies $(\mathrm{G})_{\mathcal{P}, \kappa}$.}

Proof. Let $\left\langle b_{\alpha}: \alpha<\kappa\right\rangle$ be an increasing sequence of members of $\mathcal{P}$, and set $b=\bigcup_{\alpha<\kappa} b_{\alpha}$. Apply $(\mathrm{F})_{\mathcal{P}, \kappa}$ to the set $x=\left\{b_{\alpha} \mid \alpha<\kappa\right\} \in[\mathcal{P}]^{\kappa}$, and obtain a $W$-filtered set $A \subseteq \mathcal{P}$ such that $|x \cap A|=\kappa=|A|$. Write $A=\left\{a_{i} \mid i<\kappa\right\}$, and define $a=\bigcup A$. We will show that $b \subseteq a$ and $a$ is $W$-filtered. By Lemma 3.1 this implies that $b$ is $W$-filtered, as required.

Since $x \cap A$ contains unboundedly many $b_{\alpha}$ 's, we have $b \subseteq a=\bigcup A$. Since $A$ is $W$-filtered, $\left\{\delta<\kappa \mid\left\{a_{i} \mid i<\delta\right\} \in W\right\}$ contains a club set, and hence the larger set

$$
C=\left\{\delta<\kappa \mid \bigcup_{i<\delta} a_{i} \in W\right\}
$$

contains a club set. This gives a witness that $a$ is $W$-filtered.

3.4. Remark. If $(\mathcal{P}, \subseteq)$ is cofinal in $\left(\lambda^{<\kappa}, \subseteq\right)$, Theorem 3.3 and Lemma 3.2 imply that the strong covering property for sequences of subsets of $\lambda$ follows from $(\mathrm{F})_{\mathcal{P}, \kappa}$. The theorem also shows in this case that $(\mathrm{F})_{\mathcal{P}, \kappa}$ implies that every $x \in[\lambda]^{\kappa}$ is contained in a $W$-filtered set.

3.5. Remark. We could have replaced the club filter on $\kappa$ by any filter $\mathbb{D}$ over $\kappa$ containing the club sets. Then a $W$-DD-filtered set would be a set $A$ with a decomposition $A=\bigcup_{i<\kappa} a_{i}$ such that $\left\{\delta<\kappa: \bigcup_{i<\delta} a_{i} \in W\right\} \in \mathbb{D}$. The statement $(\mathrm{F})_{\lambda}$ would be replaced by (the weaker) $(\mathrm{F})_{\mathbb{D}}$, and correspondingly $(\mathrm{G})_{\lambda}$ would be replaced by $(\mathrm{G})_{\mathbb{D}}$. The proof that $(\mathrm{F})_{\mathbb{D}} \Rightarrow(\mathrm{G})_{\mathbb{D}}$ would be almost the same if " $X$ is a club" is replaced by $X \in \mathbb{D}$. Note: working with an arbitrary normal filter $\mathbb{D}$ on $\kappa$ does not materially change the proof, but gives a much weaker assumption. 
4. How to derive $(\mathrm{F})$. We saw in Remark 3.4 that $(\mathrm{F})$ is a form of strong covering. In this section we see how to derive it from some other putatively weaker assumptions.

We start by listing the necessary assumptions.

$(\mathrm{H})_{\kappa} \quad$ Every ordinal $\alpha$ in the interval $\left(\kappa, \kappa^{+}\right)$is $W$-filtered.

4.1. REMARK. Notice that $H_{\kappa}$ is a weakening of the assumption that $\kappa_{V}^{+}=\kappa_{W}^{+}$. It is properly weaker, as can be seen by starting with a model $W$ and $\kappa^{+}<\lambda$ with $\kappa, \lambda$ both regular in $W$, and doing the Levy collapse $\operatorname{Coll}(\kappa,<\lambda)$.

Recall that a function $g: A \rightarrow \mathrm{ON}$ from a set of ordinals $A$ of size $\kappa$ into the ordinals is $W$-filtered iff there exists a filtration $\vec{a}$ of $A$ such that for all $i$ both $a_{i}$ and $g\left\lceil a_{i}\right.$ are in $W$.

$(\mathrm{J})_{\kappa, \lambda}^{\prime} \quad$ For any $\mathfrak{a} \subseteq \mathrm{REG}^{W} \cap\left(\lambda \backslash \kappa^{+}\right)$of cardinality $\kappa$, if $\mathfrak{a}$ is $W$-filtered, then for every $f \in \prod \mathfrak{a}$ there exists $g \in \prod \mathfrak{a}$ such that $f \leq g$ and $g$ is $W$-filtered.

Here $f \in \prod \mathfrak{a}$ means that $f(\alpha) \in \alpha$ for every $\alpha \in \mathfrak{a}$, and $f \leq g$ means that $(\forall \alpha \in \mathfrak{a})(f(\alpha) \leq g(\alpha)) . \quad J^{\prime}$ is an apparently weaker version of the property $J$ defined in the introduction; actually it is equivalent.

$(\mathrm{B})_{\kappa^{+}, \lambda}^{-} \quad$ For every ordinal $\alpha \in \lambda$, if $\operatorname{cf}(\alpha)<\kappa^{+}$, then $\operatorname{cf}_{W}(\alpha)<\kappa_{V}^{+}$.

4.2. REMARK. The hypotheses $(\mathrm{B})_{\kappa^{+}, \lambda}^{-}$and $(\mathrm{J})_{\kappa, \lambda}^{\prime}$ are both consequences of $(\mathrm{B})_{\kappa^{+}, \lambda}$.

4.3. Theorem. If $(\mathrm{B})_{\kappa^{+}, \lambda}^{-},(\mathrm{J})_{\kappa, \lambda}^{\prime}$ and $(\mathrm{H})_{\kappa}$ then $(\mathrm{F})_{\lambda, \kappa}$.

Proof. Let $x \in[\lambda]^{\kappa}$ be given; we shall find a $W$-filtered set $A$ such that $|x \cap A|=\kappa$. For this we define by induction $W$-filtered sets $N_{n}$ for each $n<\omega$ such that $x \subseteq \bigcup_{n<\omega} N_{n}$ and then necessarily some $N_{i}$ satisfies $\left|x \cap N_{i}\right|=\kappa$ and is thus as required.

First we fix a rich enough first-order structure $\mathfrak{B} \in W$, with universe $\lambda$ and finitely many functions and relations. The meaning of "rich enough" will become clear during the proof, where we will list a finite set of functions which should appear among the functions of $\mathfrak{B}$ including $x+1$.

Then we construct elementary submodels $N_{n}, M_{n} \prec \mathfrak{B}$ of size $\kappa$ such that:

(1) $N_{n}$ is $W$-filtered and $\kappa \subseteq N_{n} \subseteq N_{n+1}$.

(2) $x \subseteq M_{0}, M_{n} \subseteq M_{n+1}$ ( $x$ is the given set).

(3) $N_{n} \subseteq M_{n}$; also $\sup \left(M_{n}\right) \leq \sup \left(N_{n+1}\right)$.

(4) For $\mathfrak{a}_{n}=\mathrm{REG}^{W} \cap\left(\lambda \backslash \kappa^{+}\right) \cap N_{n}$, if we define $f_{n}=\mathrm{Ch}_{\mathfrak{a}_{n}}^{M_{n}}$ and $g_{n}=\mathrm{Ch}_{\mathfrak{a}_{n}}^{N_{n+1}}$, then $f_{n} \leq g_{n}$. 
Recall that $\mathrm{Ch}_{A}^{M}(\theta)=\sup (M \cap \theta)$ for $\theta \in A$. Thus $f_{n} \leq g_{n}$ means simply that $\sup \left(M_{n} \cap \theta\right) \leq \sup \left(N_{n+1} \cap \theta\right)$ for all $W$-regular $\theta \geq \kappa^{+}$in $N_{n}$.

The construction begins by setting $N_{0}=\operatorname{Sk}(\kappa)$, where $\operatorname{Sk}(X)$ is the Skolem closure of $X \subseteq \lambda$ in $\mathfrak{B}$. This maintains the induction hypotheses because of the following fact.

4.4. Lemma. If $X$ is $W$-filtered, then so is $\operatorname{Sk}(X)$.

P r o of. Easy, using the fact that $\mathfrak{B} \in W$.

In the second step, $M_{0}$ is defined to be any elementary submodel of $\mathfrak{B}$ of size $\kappa$ which contains $x$ and $N_{0}$. For example, $M_{0}=\operatorname{Sk}\left(N_{0} \cup x\right)$ will do.

Suppose now that $N_{n}$ and $M_{n}$ have been defined. We first define $N_{n+1}$ as follows. Set $\mathfrak{a}_{n}=\operatorname{REG}^{W} \cap\left(\lambda \backslash \kappa^{+}\right) \cap N_{n}$. For every $\theta \in \mathfrak{a}_{n}$, as $(\mathrm{B})_{\kappa^{+}, \lambda}^{-}$holds and as $\theta \geq \kappa^{+}$is regular in $W, \operatorname{cf}_{V}(\theta) \geq \kappa^{+}$. Hence $f_{n}(\theta)=\sup \left(M_{n} \cap \theta\right)$ $<\theta$, and the function $f_{n}$ thus defined is in $\prod \mathfrak{a}_{n}$. So $(J)_{\kappa, \lambda}^{\prime}$ can be applied to $\mathfrak{a}_{n}, f_{n}$ and there exists $g_{n} \in \prod \mathfrak{a}_{n}$ which is $W$-filtered and such that $f_{n} \leq g_{n}$. It follows that the set $\left\{g_{n}(\theta): \theta \in \mathfrak{a}_{n}\right\}$ is also $W$-filtered. Let $\gamma_{n}=\sup \left(M_{n} \cap \kappa^{+}\right)$; then $\gamma_{n}$ is $W$-filtered, because we are assuming $(\mathrm{H})_{\kappa}$. Let $\sigma_{n}=\sup M_{n}$ if $\sup M_{n}<\lambda$, and $\sigma_{n}=0$ otherwise. Define

$$
N_{n+1}=\operatorname{Sk}\left(N_{n} \cup\left\{g_{n}(\theta): \theta \in \mathfrak{a}_{n}\right\} \cup \gamma_{n} \cup\left\{\sigma_{n}\right\}\right) .
$$

Then $N_{n+1}$ is $W$-filtered. Finally, define

$$
M_{n+1}=\operatorname{Sk}\left(N_{n+1} \cup M_{n}\right) .
$$

This ends the inductive definition of the models, and we now prove that $\bigcup_{n<\omega} N_{n}=\bigcup_{n<\omega} M_{n}$. This obviously implies $x \subseteq \bigcup_{n<\omega} M_{n}$ and thus ends the proof.

Let $N=\bigcup_{n<\omega} N_{n}, M=\bigcup_{n<\omega} M_{n}$. The following properties easily follow from our construction:

(1) $N \prec M$ (since $N_{n} \prec M_{n}$ ).

(2) $N \cap \kappa^{+}=M \cap \kappa^{+}=\sup \left\{\gamma_{n}: n<\omega\right\}$.

(3) $\sup N=\sup M \leq \lambda$.

(4) For every $\theta \in N \cap \mathrm{REG}^{W} \backslash \kappa^{+}, \sup (N \cap \theta)=\sup (M \cap \theta)$. Indeed, $\sup (N \cap \theta) \leq \sup (M \cap \theta)$ follows from $N \subseteq M$, and the other direction of the inequality follows from $\sup \left(M_{n} \cap \theta\right) \leq \sup \left(N_{n+1} \cap \theta\right)$.

We claim that (1)-(4) imply that $M=N$. To see this, assume otherwise and then $M \backslash N \neq \emptyset$. Let $\alpha=\min (M \backslash N)$. By (3), $\beta=\min (N \backslash \alpha)$ is defined. We shall derive a contradiction in the following complete analysis of cases for $\beta$.

CASE: $\beta<\kappa^{+}$. Then $\alpha<\kappa^{+}$and this contradicts (2). 
CASE: $\beta$ is a successor ordinal. Let $\tau$ be such that $\tau+1=\beta$. Then $\tau \in N$ if $\mathfrak{B}$ is closed under predecessors. We will therefore demand that the predecessor function appear among the functions of $\mathfrak{B}$.

Now $\alpha<\beta$ implies $\alpha \leq \tau$, and yet $\alpha=\tau$ is not possible since $\alpha \notin N$; hence $\alpha<\tau<\beta$ contradicts the minimality of $\beta$.

CASE: $\beta$ is singular in $W$. Let $\tau=\operatorname{cf}^{W}(\beta)$. Then $\tau \in N$ as long as the $W$-cofinality function is in $\mathfrak{B}$. We therefore demand that $\mathrm{cf}_{W}$ is among the functions of $W$.

Now as $\tau<\beta$ ( $\beta$ being singular), $\tau<\alpha$ follows. We will demand that $\mathfrak{B}$ contains a function $\mathrm{COF}$ which assigns to every $W$-singular cardinal $\zeta$ a cofinal sequence $\operatorname{COF}(\zeta) \in W$ of length $\operatorname{cf}_{W}(\zeta)$. Then $\operatorname{COF}(\beta): \tau \rightarrow \beta$ is cofinal in $\beta$ and so, as $\alpha \in M$, there is $\zeta<\tau$ in $M$ such that $\alpha^{\prime}=$ $\operatorname{COF}(\beta)(\zeta) \geq \alpha$. Since $N \cap \alpha=M \cap \alpha$ by the minimality of $\alpha, \zeta \in N$. Hence $\alpha^{\prime} \in N$ is an ordinal in $[\alpha, \beta)$, in contradiction to the minimality of $\beta$.

CASE: $\beta$ is regular in $W$. This is the last case. We know by (4) that $\sup (N \cap \beta)=\sup (M \cap \beta)$. Hence $\sup (N \cap \beta) \geq \alpha$, which is again a contradiction to $\beta=\min (N \backslash \alpha)$.

This concludes the proof of the Theorem.

We end this section by considering a variation $(J)_{\lambda}^{\prime \prime}$ which seems weaker than $(J)_{\lambda}^{\prime}$ but suffices for Theorem 4.3:

$(\mathrm{J})_{\lambda}^{\prime \prime} \quad$ If $A \subseteq \mathrm{REG}^{W} \cap\left(\lambda \backslash \kappa^{+}\right)$of cardinality $\kappa$ is $W$-filtered and $f \in \prod A$, then there exists $i^{*}<\kappa$ and a collection $\left\{f_{i}: i<i^{*}\right\}, f_{i} \in \prod A$, such that $f \leq \sup \left\{f_{i}: i<i^{*}\right\}$ and each $f_{i}$ is $W$-filtered.

4.5. Theorem. Theorem 4.3 still holds if $(\mathrm{J})_{\lambda}^{\prime \prime}$ replaces $(\mathrm{J})_{\lambda}^{\prime}$.

Proof. Given $i^{*}<\kappa$, let us say that $A$ is $\left(i^{*}, W\right)$-filtered iff $A$ is a union of $i^{*}$ sets each of which is $W$-filtered.

We will define $N_{n}, M_{n}$ as before, but require now that $N_{n}$ is $\left(i_{n}^{*}, W\right)$ filtered for some $i_{n}^{*}<\kappa$. The construction is very similar, but in defining $N_{n+1}$ it is not a single function that is added to $N_{n}$, but rather $i_{n}^{*}$ functions, each $W$-filtered. Finally $N=M$ as before, and for some $n$ we have $\left|N_{n} \cap x\right|=\kappa$.

As $N_{n}$ is $\left(i_{n}^{*}, W\right)$-filtered, we may find a $W$-filtered set $X \subseteq N_{n}$ such that $|X \cap x|=\kappa$ as required.

5. The pcf induction lemma. The results in the last section indicate the usefulness of the hypothesis $(\mathrm{J})^{\prime}$. In this section we prove a crucial lemma, which we will exploit in the final section of the paper to prove that two rather different sets of assumptions will each imply $(\mathrm{J})^{\prime}$. 
5.1. Lemma. Let $W \subseteq V$ be two transitive class models of set theory. In $V$ let $\kappa$ be regular, with $\kappa<\sigma \in \operatorname{Card}_{W}$, and $H=H_{\sigma}=\{x \in V$ : the transition closure of $x$ has cardinality $<|\sigma|_{V}^{+}$and rank $\left.<\sigma\right\}$. Assume that:

(1) $N \prec\left(H_{\sigma}, \in,<^{*}, W \cap H_{\sigma}\right)$ where $<^{*}$ is a wellordering of $H_{\sigma}$.

(2) $|N|=\kappa, \kappa \subseteq N$.

(3) If $\tau \in N \cap \mathrm{REG}^{W}$ with $\tau \geq \kappa_{V}^{+}$, then

(a) $\sup (N \cap \tau)<\tau$.

(b) There exists an unbounded subset $C$ of $N \cap \tau$ with $C \in W$.

(4) For all $\mathfrak{a} \subseteq \mathrm{REG}^{W} \backslash \kappa_{V}^{+}$with $\mathfrak{a} \in N \cap W,|\mathfrak{a}|<\kappa$ and $\max \left(\operatorname{pcf}_{W}(\mathfrak{a})\right)$ $<\sigma$, if $h \in \prod \mathfrak{a}$ and $h(\varrho)<\sup (N \cap \varrho)$ for all $\varrho \in \mathfrak{a}$, then there is $g \in$ $\prod \mathfrak{a} \cap N \cap W$ such that $h(\varrho)<g(\varrho)$ for all $\varrho \in \mathfrak{a}$.

Then for all $\mathfrak{a} \subseteq \mathrm{REG}^{W} \backslash \kappa_{V}^{+}$with $\mathfrak{a} \in N \cap W, \max \left(\operatorname{pcf}_{W}(\mathfrak{a})\right)<\sigma$ and $|\mathfrak{a}|<\kappa$, if we define $\operatorname{ch}_{\mathfrak{a}}^{N}: \varrho \in \mathfrak{a} \mapsto \sup (N \cap \varrho)$ then $\operatorname{ch}_{\mathfrak{a}}^{N} \in W$.

Proof. Fix a structure $N$ with these properties. We prove the lemma by induction on $\theta=\max \left(\operatorname{pcf}_{W}(\mathfrak{a})\right)$. We start the induction by observing that if $\theta=\kappa_{V}^{+}$then necessarily $\mathfrak{a}=\left\{\kappa_{V}^{+}\right\}$(because $\mathfrak{a} \subseteq \operatorname{pcf}(\mathfrak{a})$ and $\min (\mathfrak{a})=\min (\operatorname{pcf}(\mathfrak{a})))$ and the result is trivial.

For the general case, start by observing that since $\mathfrak{a} \in N$ and $\theta<\sigma$ and due to the appearance of $W \cap H_{\sigma}$ in assumption (1), we have $\theta \in N$. Notice also that by (3)(a) we have $\operatorname{ch}_{\mathfrak{a}}^{N} \in \prod \mathfrak{a}$.

Fix a sequence $\left\langle f_{\gamma}: \gamma\langle\theta\rangle \in N \cap W\right.$ such that

$$
W \models " \vec{f} \text { is increasing cofinal in } \prod \mathfrak{a} / J_{<\theta} " .
$$

Such a sequence $\vec{f}$ exists in $W$ by a basic fact from pcf theory (see Theorem 8.7 from the Appendix) and since $\mathfrak{a}, \theta \in N$ we may assume that $\vec{f}$ lies in $N$.

Now $\theta \in N$ and $\theta$ is regular in $W$, so by (3)(b) we may fix some $C$ unbounded in $N \cap \theta$ with $C \in W$. Define $f(\varrho)=\sup _{\gamma \in C} f_{\gamma}(\varrho)$ for $\varrho \in \mathfrak{a}$. Then:

(1) $f \in W$ because $f$ is defined from $\vec{f}, \mathfrak{a}$ and $C$, which are all in $W$.

(2) For each $\gamma \in C$ and $\varrho \in \mathfrak{a}, f_{\gamma}(\varrho) \in N \cap \varrho$ because $C, \mathfrak{a} \subseteq N$ and $\vec{f} \in N$. Therefore $f(\varrho) \leq \operatorname{ch}_{\mathfrak{a}}^{N}(\varrho)<\varrho$.

Let $\mathfrak{b}:=\left\{\varrho \in \mathfrak{a} \mid f(\varrho)<\operatorname{ch}_{\mathfrak{a}}^{N}(\varrho)\right\}$, and define $h$ by setting $h \uparrow \mathfrak{b}=f \uparrow \mathfrak{b}$ and $h \uparrow(\mathfrak{a} \backslash \mathfrak{b})$ to be the zero function. By (4) we may find $g \in \prod \mathfrak{a} \cap N \cap W$ such that $h<g$, so that $(\forall \varrho \in \mathfrak{b})(f(\varrho)<g(\varrho))$. By elementarity there is $\gamma \in N \cap \theta$ such that $g<_{J_{<\theta}} f_{\gamma}$, and since $C$ is unbounded in $N \cap \theta$ we may assume that $\gamma \in C$. By the construction of $f, f_{\gamma} \leq f$.

Now let $\mathfrak{b}^{*}=\left\{\varrho \in \mathfrak{a} \mid f_{\gamma}(\varrho) \leq g(\varrho)\right\}$. Then $\mathfrak{b}^{*} \in N \cap W$ because the parameters $\mathfrak{a}, f_{\gamma}$ and $g$ lie in $N \cap W$. Moreover $\mathfrak{b}^{*} \in J_{<\theta}$, because $g<_{J_{<\theta}} f_{\gamma}$. Finally $\mathfrak{b} \subseteq \mathfrak{b}^{*}$, because $\varrho \in \mathfrak{b} \Rightarrow f_{\gamma}(\varrho) \leq f(\varrho)<g(\varrho)$. 
But now $\max \left(\operatorname{pcf}_{W}\left(\mathfrak{b}^{*}\right)\right)<\theta$, because otherwise in $W$ we could find an ultrafilter $D$ on $\mathfrak{b}^{*}$ with $\operatorname{cf}\left(\prod \mathfrak{b}^{*} / D\right) \geq \theta$. This contradicts $\mathfrak{b}^{*} \in J_{<\theta}$.

Applying the induction hypothesis gives $\operatorname{ch}_{\mathfrak{b}^{*}}^{N} \in W$. Therefore

$$
\operatorname{ch}_{\mathfrak{a}}^{N}=f \uparrow\left(\mathfrak{a} \backslash \mathfrak{b}^{*}\right) \cup \operatorname{ch}_{\mathfrak{b}^{*}}^{N} \in W,
$$

and we are done.

We conclude this section by proving that structures $N$ with most of the properties demanded in Lemma 5.1 are quite easily manufactured.

5.2. Lemma. Let $W \subseteq V$ be two transitive class models of set theory. In $V$ let $\kappa$ be regular, with $\kappa<\sigma \in \operatorname{Card}_{W}$. Assume that $\kappa$-covering holds between $V$ and $W$, and that $\operatorname{cf}_{W}(\alpha) \geq \kappa_{V}^{+} \Rightarrow \operatorname{cf}_{V}(\alpha) \geq \kappa_{V}^{+}$for all $\alpha<\sigma$. Let $\mathcal{M}=\left(H_{\sigma}, \in,<^{*}, W \cap H_{\sigma}\right)$ where $<^{*}$ is a wellordering of $H_{\sigma}$. Let $\left\langle N_{i}: i<\beta\right\rangle$ be an increasing and continuous chain of elementary substructures of $\mathcal{M}$ such that:

(1) $\kappa \subseteq N_{0}$.

(2) $\beta<\kappa^{+}$and $\operatorname{cf}(\beta)=\kappa$.

(3) $\left|N_{i}\right|=\kappa$ for all $i<\beta$.

(4) $\left\langle N_{i}: i \leq j\right\rangle \in N_{j+1}$ for all $j<\beta$.

Let $N:=\bigcup_{i<\beta} N_{i}$. Then

(1) If $\theta \in N \cap \mathrm{REG}^{W}$ with $\theta \geq \kappa_{V}^{+}$, then

(a) $\sup (N \cap \theta)<\theta$.

(b) $\operatorname{cf}_{V}(\sup (N \cap \theta))=\kappa$.

(2) For all $\mathfrak{a} \subseteq \mathrm{REG}^{W} \backslash \kappa_{V}^{+}$with $\mathfrak{a} \in N \cap W$ and $|\mathfrak{a}|<\kappa$, if $g \in \prod \mathfrak{a}$ and $g(\varrho)<\sup (N \cap \varrho)$ for all $\varrho \in \mathfrak{a}$, then there is $h \in \prod \mathfrak{a} \cap N \cap W$ such that $g(\varrho)<h(\varrho)$ for all $\varrho \in \mathfrak{a}$.

Proof. We take each claim in turn. Since $\theta \geq \kappa_{W}^{+}$and $\theta \in \mathrm{REG}^{W}$, it follows from our assumptions that $\mathrm{cf}_{V}(\theta)>\kappa$. As $|N|=\kappa$, it follows that $\sup (N \cap \theta)<\theta$ and we have proved claim (1)(a) for every $\varrho \in \mathfrak{a}$. Since $\theta \in N, \theta \in N_{i}$ for some $i<\beta$. For all $j>i$ we have $\theta, N_{i} \in N_{j}$, so that $\sup \left(N_{i} \cap \theta\right) \in N_{j}$ and therefore $\sup \left(N_{i} \cap \theta\right)<\sup \left(N_{j} \cap \theta\right)$. The sequence $\left\langle\sup \left(N_{j} \cap \theta\right): i<j<\beta\right\rangle$ is increasing and cofinal in $\sup (N \cap \theta)$ so $\operatorname{cf}(\sup (N \cap \theta))=\operatorname{cf}(\beta)=\kappa$. This proves claim (1)(b).

Let $\mathfrak{a}, g$ be as in claim (2) of the lemma. If $\mathfrak{a} \in N_{i}$, a similar argument to that given in the last paragraph shows that for every $\varrho \in \mathfrak{a}$ the sequence $\left\langle\sup \left(N_{j} \cap \varrho\right): i<j<\beta\right\rangle$ is increasing and cofinal in $\sup (N \cap \varrho)$. Hence for every $\varrho \in \mathfrak{a}$ there is $j<\beta$ with $g(\varrho)<\sup \left(N_{j} \cap \varrho\right)$; since $\operatorname{cf}(\beta)=\kappa>|\mathfrak{a}|$ we may find a fixed $j$ such that $g(\varrho)<\sup \left(N_{j} \cap \varrho\right)$ for every $\varrho \in \mathfrak{a}$. Since $N_{j} \in N$, the function $g^{*}: \varrho \mapsto \sup \left(N_{j} \cap \varrho\right) \in N$. 
Now we apply covering in a routine way to find a set $X \subseteq \mathfrak{a} \times \bigcup \mathfrak{a}$ with $|X|_{V}<\kappa, X \in W$ and $X \supseteq g^{*}$. Since $g^{*} \in N$ and $N \prec \mathcal{M}$ (a structure into which we built information about $W$ ) we may assume that $X \in N \cap W$. We define $h(\varrho)=\sup \{\beta \mid(\varrho, \beta) \in X\}$, and then $h \in N \cap W$ and also, since $\operatorname{cf}_{V}(\varrho)>\kappa, h \in \prod \mathfrak{a}$. Clearly $g(\varrho)<g^{*}(\varrho) \leq h(\varrho)$ for all $\varrho$, and we are done.

So the missing ingredient for applying Lemma 5.1 is the existence of a set $C$ with $C \in W$ and $C$ unbounded in $N \cap \theta$. In the next section we see two ways of guaranteeing the existence of such a $C$.

Notice that the covering assumption in Lemma 5.2 can be weakened. All we need is that a set of size less than $\kappa$ can be covered by a set of size less than $\kappa^{+}$.

6. Applying the pcf induction lemma. We begin by showing that we can use Lemma 5.1 to prove instances of the principle $(\mathrm{J})^{\prime}$ which we now recall.

$(\mathrm{J})_{\kappa, \lambda}^{\prime} \quad$ For any $\mathfrak{a} \subseteq \mathrm{REG}^{W} \cap\left(\lambda \backslash \kappa^{+}\right)$of cardinality $\kappa$, if $\mathfrak{a}$ is $W$-filtered, then for every $f \in \prod \mathfrak{a}$ there exists $g \in \prod \mathfrak{a}$ such that $f \leq g$ and $g$ is $W$-filtered.

As usual, $W$ and $V$ are transitive class models of ZFC with $W \subseteq V$, and $\kappa$ is a regular uncountable cardinal in $V$.

6.1. Lemma. Let $\mathfrak{a} \subseteq \mathrm{REG}^{W} \backslash \kappa_{V}^{+}$be a $W$-filtered set of size $\kappa$, as witnessed by a filtration $\vec{a}$ such that $a_{i} \in W$ for all $i<\kappa$. Let $f \in \prod \mathfrak{a}$. Suppose that $f, \mathfrak{a}, \vec{a} \in N$ where $N$ is a structure obeying the assumption of Lemma 5.1 , and such that $\max \left(\operatorname{pcf}_{W}\left(a_{i}\right)\right) \in N$ for all $i$. Then $\operatorname{ch}_{\mathfrak{a}}^{N}$ is $W$-filtered and $f \leq \operatorname{ch}_{\mathfrak{a}}^{N}$.

Proof. Since $\mathfrak{a} \subseteq N$ (because $|\mathfrak{a}|=\kappa \subseteq N$ ) it is easy to see that $f \leq \operatorname{ch}_{\mathfrak{a}}^{N}$. Since we built the filtration $\vec{a}$ into $N$ we see that for all $i<\kappa$ we have $a_{i} \in N \cap W$, and we may apply Lemma 5.1 to conclude that $\operatorname{ch}_{a_{i}}^{N} \in W$. Therefore $\left\langle\operatorname{ch}_{a_{i}}^{N}: i<\kappa\right\rangle$ gives a filtration of $\operatorname{ch}_{\mathfrak{a}}^{N}$ and we are done.

6.2. Remark. The same idea could be used to derive the stronger property $(\mathrm{J})$ defined in the introduction. That is, the assumption that $\vec{a}$ is increasing is never used.

Now we describe, in Lemmas 6.4 and 6.6, two ways of building structures $N$ that satisfy the hypotheses of Lemma 5.1.

6.3. Definition. Let $\mu$ and $\theta$ be regular cardinals with $\mu<\theta$. Then a square sequence on $\theta$ for cofinalities less than $\mu$ is a sequence $\left\langle C_{\alpha}: \alpha<\theta\right.$, 
$\operatorname{cf}(\alpha)<\mu\rangle$ such that $C_{\alpha}$ is club in $\alpha$, o.t. $\left(C_{\alpha}\right)<\mu$ and $\beta \in \lim \left(C_{\alpha}\right) \Rightarrow C_{\beta}=$ $C_{\alpha} \cap \beta$.

6.4. Lemma. Let $W$ and $V$ be two transitive class models of ZFC with $W \subseteq V$. Let $\kappa>\aleph_{0}$ be regular in $V$ and $\kappa<\sigma \in \operatorname{Card}_{W}$. Suppose that

$W \models$ "there is a square on $\theta$ for cofinalities less than $\kappa_{V}^{+}$"

for every $W$-regular cardinal $\theta<\sigma$. Suppose also $\operatorname{cf}_{W}(\alpha) \geq \kappa_{V}^{+} \Rightarrow \operatorname{cf}_{V}(\alpha) \geq$ $\kappa_{V}^{+}$for all $\alpha<\sigma$. Let $\left\langle N_{i}: i<\beta\right\rangle$ be a sequence of substructures of $\left(H_{\sigma}, \in,<^{*}, W \cap H_{\sigma}\right)$ such that:

(1) $\operatorname{cf}(\beta)>\omega$ and $\beta<\kappa^{+}$.

(2) $\kappa \subseteq N_{0}$ and $\left|N_{i}\right|=\kappa$.

(3) $\left\langle N_{i}: i \leq j\right\rangle \in N_{j+1}$ for all $j<\beta$.

Let $N=\bigcup_{i<\beta} N_{i}$, and let $\theta \in \mathrm{REG}^{W} \cap N$ with $\theta \geq \kappa_{V}^{+}$. If $\vec{C} \in W$ is a square on $\theta$ for cofinalities less than $\kappa_{V}^{+}$then $C_{\sup (N \cap \theta)} \subseteq N$.

Proof. Let $\bar{\theta}:=\sup (N \cap \theta)$, where $\bar{\theta}<\theta$ because $|N|=\kappa$ and $\operatorname{cf}_{V}(\theta)>\kappa$. Then $C_{\bar{\theta}}$ is defined because $\operatorname{cf}_{W}(\bar{\theta})<\kappa_{V}^{+}$.

If $\theta \in N_{i}$ and we define $\theta_{j}=\sup \left(N_{j} \cap \theta\right)$ then we may argue as in Lemma 5.1 that $\left\langle\theta_{j}: i \leq j<\beta\right\rangle$ is increasing, continuous and cofinal in $\bar{\theta}$. Now since $\operatorname{cf}(\beta)>\omega$ and $C_{\bar{\theta}}$ is club in $\bar{\theta}$ there is a club $D$ of $j<\beta$ such that $\theta_{j} \in \lim \left(C_{\bar{\theta}}\right)$. For each such $j, C_{\theta_{j}}=C_{\bar{\theta}} \cap \theta_{j}$; since $\theta_{j} \in N,\left|C_{\theta_{j}}\right| \leq \kappa$ and $\kappa \subseteq N$ we see that $C_{\theta_{j}} \subseteq N$, so $C_{\bar{\theta}}=\bigcup_{j \in D} C_{\theta_{j}} \subseteq N$ and we are done.

6.5. Remark. By Lemmas 5.2 and 6.4, the structure $N$ defined in Lemma 5.2 obeys all the hypotheses of Lemma 5.1.

We now describe another way of getting the hypotheses of Lemma 5.1 to hold. Here we drop the assumption of squares but pay for this by needing to assume more resemblance between $V$ and $W$.

6.6. Lemma. Let $W, V$ be transitive class models of $Z F C$ with $W \subseteq V$. Let $\kappa$ be regular in $V$ with $\aleph_{0}<\kappa<\sigma \in \operatorname{Card}_{W}$ and let $\mathcal{M}=\left(H_{\sigma}, \in,<^{*}\right.$, $\left.W \cap H_{\sigma}\right)$ for some wellordering $<^{*}$ of $H_{\sigma}$. Assume that $\kappa_{V}^{+}=\kappa_{W}^{+}, \kappa_{V}^{++}=$ $\kappa_{W}^{++}, \operatorname{cf}_{W}(\tau) \geq \kappa^{+} \Rightarrow \operatorname{cf}_{V}(\tau) \geq \kappa^{+}, \operatorname{cf}_{W}(\tau) \geq \kappa^{++} \Rightarrow \operatorname{cf}_{V}(\tau) \geq \kappa^{++}$for every $\tau<\sigma$. Let $\left\langle N_{i}: i<\kappa^{+}\right\rangle$be a continuous and increasing chain of elementary submodels of $\mathcal{M}$ such that $\kappa \subseteq N_{0},\left|N_{j}\right|=\kappa,\left\langle N_{i}: i \leq j\right\rangle \in N_{j+1}$ for all $j$. Then there exists $j$ such that for every $\theta \in N_{j}$ with $\theta \in \mathrm{REG}^{W}$, $\theta \geq \kappa^{+}$there is $C \in W$ such that $C$ is unbounded in $N_{j} \cap \theta$ and $\operatorname{cf}(j)=\kappa$.

Proof. First we dismiss the case $\theta=\kappa^{+}$. In this case there is not too much to prove because $N_{j} \cap \theta \in \theta$ for any $j$. So henceforth we assume that $\theta \geq \kappa^{++}$. 
Next we claim that for a fixed $\theta \in N:=\bigcup_{j} N_{j}$, where $\theta \in \mathrm{REG}^{W} \backslash \kappa^{++}$, there is a club $D$ in $\kappa^{+}$such that every $j \in D$ with $\operatorname{cf}(j)=\kappa$ has the property claimed.

Let $\theta \in N_{i}$, and fix $\left\langle E_{\beta}: \beta<\theta\right\rangle \in W \cap N_{i}$ such that $E_{\beta}$ is club in $\beta$ and o.t. $\left(E_{\beta}\right)=\operatorname{cf}_{W}(\beta)$. Let $\theta_{j}:=\sup \left(\theta \cap N_{j}\right)$, so that as usual $\left\langle\theta_{j}: i \leq j<\kappa^{+}\right\rangle$ is continuous increasing and cofinal in $N \cap \theta$. Our assumptions imply that $\operatorname{cf}_{V}(\theta) \geq \kappa^{++}$, so that in particular $\bar{\theta}:=\sup (N \cap \theta)<\theta$ and $E_{\bar{\theta}}$ is defined. Our assumptions also imply that $\operatorname{cf}_{W}(\bar{\theta})=\operatorname{cf}_{V}(\bar{\theta})=\kappa^{+}$, so that o.t. $\left(E_{\bar{\theta}}\right)=\kappa^{+}$.

Now define $D \subseteq \kappa^{+}$by

$$
D=\left\{j>i \mid E_{\bar{\theta}} \cap \theta_{j} \cap N \subseteq N_{j}, \theta_{j} \in \lim \left(E_{\bar{\theta}}\right)\right\} .
$$

It is easy to see that $D$ is club in $\kappa^{+}$, the key point being that o.t. $\left(E_{\bar{\theta}}\right)=\kappa^{+}$ so that for each $j$ there is $\zeta<\kappa^{+}$with $E_{\bar{\theta}} \cap \theta_{j} \cap N \subseteq N_{\zeta}$.

Let $j \in D$ with $\operatorname{cf}(j)=\kappa$, and let $C=E_{\theta_{j}} \cap E_{\bar{\theta}}$. Then $C \in W$ because $C$ is the intersection of two sets in $W$. By our assumptions again o.t. $\left(E_{\theta_{j}}\right)=\operatorname{cf}_{W}\left(\theta_{j}\right)=\operatorname{cf}_{V}\left(\theta_{j}\right)=\kappa$, so easily o.t. $(C)=\kappa$. Since $\vec{E} \in N_{i}$, $\kappa \subseteq N_{0}$ and $\theta_{j} \in N_{j+1}$ we see that $E_{\theta_{j}} \subseteq N_{j+1} \subseteq N$. Now the key point is that

$$
C=E_{\bar{\theta}} \cap E_{\theta_{j}} \subseteq E_{\bar{\theta}} \cap \theta_{j} \cap N \subseteq N_{j},
$$

as required. Lastly, as both $E_{\theta_{j}}$ and $E_{\bar{\theta}}$ contain a club of $\theta_{j}$ and $\operatorname{cf}\left(\theta_{j}\right)=$ $\kappa>\aleph_{0}$, also $C$ contains a club of $\theta_{j}$, hence is unbounded in it.

To finish the argument we just take a diagonal intersection. For each appropriate $\theta$ fix $D_{\theta}$ club in $\kappa^{+}$such that every $j$ of cofinality $\kappa$ in $D_{\theta}$ is as desired. Now for each $i$ let $D_{i}^{*}=\bigcap_{\theta \in N_{i}} D_{\theta}$, and define a diagonal intersection $D^{*}=\left\{j<\kappa^{+} \mid i<j \Rightarrow j \in D_{i}^{*}\right\}$. Then if $j \in D^{*}$ with $\operatorname{cf}(j)=\kappa$, and $\theta \in N_{j}$, we see immediately that $\theta \in N_{i}$ for $i<j$ and thus $j \in D_{\theta}$.

We sum up the results of this section in a corollary using 5.1-6.6 for $\sigma_{W}^{+}$.

6.7. Corollary. Let $W \subseteq V$ be two inner models of ZFC. Suppose that $\kappa>\aleph_{0}$ is a regular cardinal in $V$ with $\kappa<\sigma \in \operatorname{Card}_{W}$. Suppose that $\operatorname{cf}_{W}(\alpha) \geq \kappa_{V}^{+} \Rightarrow \operatorname{cf}_{V}(\alpha) \geq \kappa_{V}^{+}$for all $\alpha \leq \sigma$ and $(\mathrm{I})_{\kappa, \sigma}$, and that either one of the following two assumptions holds:

(1) $W \models$ "there is a square on $\theta$ for cofinalities less than $\kappa_{V}^{+}$" for every $W$-regular $\theta$ such that $\kappa_{V}^{+} \leq \theta \leq \sigma$.

(2) $\kappa_{V}^{+}=\kappa_{W}^{+}, \kappa_{V}^{++}=\kappa_{W}^{++}$, and $\operatorname{cf}_{W}(\alpha) \geq \kappa^{++} \Rightarrow \operatorname{cf}_{V}(\alpha) \geq \kappa^{++}$for all $\alpha \leq \sigma$.

Then $(\mathrm{J})_{\kappa, \sigma}$ holds. 
7. Conclusion. We can finally state the main theorem.

7.1. Theorem. Let $W$ be an inner model of ZFC. Suppose that $\aleph_{0}<$ $\kappa<\lambda$ where $\kappa$ is regular (in $V$ ) and $\lambda$ is a cardinal in $W$. Suppose that

(1) $(\mathrm{H})_{\kappa}$ holds. That is, every ordinal in $\left(\kappa, \kappa^{+}\right)$is $W$-filtered (follows from $\left.\kappa_{V}^{+}=\kappa_{W}^{+}\right)$.

(2) There exists $\mathcal{P} \in W$ such that $W \models " \mathcal{P} \subseteq[\lambda]^{<\kappa},|\mathcal{P}|=\lambda$ " and $V \models$ "P is cofinal in $[\lambda]<\kappa "$ " this follows from e.g. "(B) $)_{\kappa, \lambda}+\left(\lambda^{\kappa}\right)_{W}=\lambda$ ".

(3) (I) $)_{\kappa, \lambda}$ holds. That is, if $W \models$ "a $\subseteq$ REG $\cap \lambda \backslash \kappa^{+},|\mathfrak{a}|<\kappa$ " then $W \models " \max (\operatorname{pcf}(\mathfrak{a})) \leq \lambda "$ (this follows from $(2)$ ).

(4) One of the following holds:

(a) $(\mathrm{D})_{\kappa_{\mathrm{V}}^{+}, \lambda}$ holds. That is, in $W$ there is a square sequence for points of cofinality less than $\kappa_{V}^{+}$on every regular cardinal in the interval $\left(\kappa_{V}^{+}, \lambda\right]$. Also $\mathrm{cf}_{W}(\tau) \geq \kappa^{+} \Rightarrow \operatorname{cf}_{V}(\tau) \geq \kappa^{+}$for all $\tau \leq \lambda$.

(b) $\kappa_{V}^{+}=\kappa_{W}^{+}, \kappa_{V}^{++}=\kappa_{W}^{++}$, and $\operatorname{cf}_{W}(\tau) \geq \kappa^{+} \Rightarrow \operatorname{cf}_{V}(\tau) \geq \kappa^{+}$, $\operatorname{cf}_{W}(\tau) \geq \kappa^{++} \Rightarrow \operatorname{cf}_{V}(\tau) \geq \kappa^{++}$for all $\tau \leq \lambda$.

Then strong $\kappa$-covering holds between $V$ and $W$, for structures with underlying set $\lambda$.

Proof. The structure of this proof can be seen by looking at the picture in the introduction. Notice that assumption (2) implies that $(\mathrm{B})_{\kappa, \lambda}$ holds. By Lemmas 6.1 and 6.4 (if we are assuming squares as in (4)(a)) or 6.1 and 6.6 (if we are assuming correctness as in $(4)(\mathrm{b})$ ) we find that $(\mathrm{J})_{\kappa, \lambda}^{\prime}$ holds. By Lemma 4.3, $(\mathrm{F})_{\lambda, \kappa}$ holds. By Theorem 3.3, $(\mathrm{G})_{\mathcal{P}, \kappa}$ holds. By Lemma 3.2 , strong $\kappa$-covering for sequences of subsets of $\lambda$ holds. As in the proof of Theorem 2.3, this implies that strong $\kappa$-covering holds for structures on $\lambda$.

7.2. Conclusion. Assume $W$ is an inner model of $V, \lambda>\kappa>\aleph_{0}, \kappa$ regular in $V, W \models$ " $\lambda=\lambda^{<\kappa}$ is a cardinal". If for $l=1,2$ we have $\kappa_{V}^{+l}=\kappa_{W}^{+l}$, and $\left[\alpha \leq \lambda \& \operatorname{cf}_{W}(\alpha) \geq \kappa^{+l} \Rightarrow \operatorname{cf}_{V}(\alpha) \geq \kappa^{+l}\right]$ then the strong $\kappa$-covering holds between $V$ and $W$ for structures with universe $\lambda$.

8. Appendix on pcf. In this appendix we will prove the elementary facts about pcf theory used in the paper. For more information see the book [Sh:g] or the survey paper [BuMa].

8.1. Definition. Let $\mathfrak{a}$ be a set of regular cardinals such that $|\mathfrak{a}|^{+}<$ $\min (\mathfrak{a})$.

(1) If $I$ is an ideal on $\mathfrak{a}$ then:

(a) If $\mathfrak{b}, \mathfrak{c} \subseteq \mathfrak{a}$, then $\mathfrak{b} \subseteq_{I} \mathfrak{c}$ iff $\mathfrak{b} \backslash \mathfrak{c} \in I$.

(b) If $f, g \in \prod \mathfrak{a}$ then $f<_{I} g$ iff $\{\theta \in \mathfrak{a} \mid g(\theta) \leq f(\theta)\} \in I$.

(2) If $F$ is a filter on $I$ and $f, g \in \prod \mathfrak{a}$ then $f<_{F} g$ iff $\{\theta \mid f(\theta)<g(\theta)\} \in F$. 
(3) A strict partial ordering $\left(\mathbb{P},<_{\mathbb{P}}\right)$ has true cofinality $\lambda$ iff $\lambda$ is regular and there exists a sequence $\left\langle p_{i}: i<\lambda\right\rangle$ such that:

(a) $i<j \Rightarrow p_{i}<\mathbb{P} p_{j}$.

(b) $(\forall p \in \mathbb{P})(\exists i<\lambda)\left(p<\mathbb{P} p_{i}\right)$.

(4) If $f, g \in \prod \mathfrak{a}$ then $f<g$ iff $f(\theta)<g(\theta)$ for all $\theta$.

In general there is no guarantee that a partial ordering will have a true cofinality. If $\mathbb{P}$ has a true cofinality it is easily seen to be unique, and we will write $\operatorname{tcf}(\mathbb{P})=\lambda$ " for the assertion where $\mathbb{P}$ has true cofinality $\lambda$; in the case where $\mathbb{P}=\left(\prod \mathfrak{a},<_{I}\right)$ for some ideal $I$ we will write $" \operatorname{tcf}\left(\prod \mathfrak{a} / I\right)=\lambda "$. The following lemma is easy, because an ultraproduct of cardinals will be linearly ordered and any linear ordering has a true cofinality.

8.2. Lemma. If $D$ is an ultrafilter on $\mathfrak{a}$ then $\left(\prod \mathfrak{a},<_{D}\right)$ has a true cofinality.

In this case we will write $" \operatorname{cf}\left(\prod \mathfrak{a} / D\right)=\lambda "$.

8.3. Definition. Let $\mathfrak{a}$ be a set of regular cardinals with $|\mathfrak{a}|^{+}<\min (\mathfrak{a})$. Then:

(1) $\operatorname{pcf}(\mathfrak{a})$ (Potential CoFinalities of $\mathfrak{a})$ is the set of regular cardinals $\lambda$ such that $\operatorname{cf}\left(\prod \mathfrak{a} / D\right)=\lambda$ for some ultrafilter $D$.

(2) If $\lambda$ is a cardinal (not necessarily regular) then $J_{<\lambda}[\mathfrak{a}]$ is the set of those $\mathfrak{b} \subseteq \mathfrak{a}$ such that $\mathfrak{b} \in D \Rightarrow \operatorname{cf}\left(\prod \mathfrak{a} / D\right)<\lambda$ for every ultrafilter $D$.

Usually $\mathfrak{a}$ will be clear from the context and we just write $J_{<\lambda}$ for $J_{<\lambda}[\mathfrak{a}]$. The following is the first key fact in pcf theory.

8.4. Lemma. Let $\mathfrak{a}, \lambda$ be as above.

(1) $J_{<\lambda}$ is an ideal (possibly an improper one).

(2) The poset $\left(\prod \mathfrak{a},<_{J_{<\lambda}}\right)$ is $\lambda$-directed, that is to say, if $F \subseteq \prod \mathfrak{a}$ and $|F|<\lambda$ then there is $g \in \prod \mathfrak{a}$ such that $(\forall f \in F)\left(f<_{J_{<\lambda}} g\right)$.

Proof. For brevity, let $J !=J_{<\lambda}$. The proof that $J$ is an ideal is fairly routine. For example let $\mathfrak{b}, \mathfrak{c} \in J$ and $\mathfrak{b} \cup \mathfrak{c} \in D$ for some ultrafilter $D$. Because $D$ is an ultrafilter, either $\mathfrak{b} \in D$ or $\mathfrak{c} \in D$, and in either case $\operatorname{cf}\left(\prod \mathfrak{a} / D\right)<\lambda$.

The proof of $\lambda$-directedness goes by induction on $|F|$ for $F \subseteq \prod \mathfrak{a}$. Let $|F|=\mu$. If $\mu \leq|\mathfrak{a}|^{+}$then we may define a bound $g(\theta)=\bigcup_{f \in F}(f(\theta)+1)$, so without loss of generality $\mu>|\mathfrak{a}|^{+}$. If $\mu$ is singular then we may write $F=\bigcup_{i<\operatorname{cf}(\mu)} F_{i}$ with $\left|F_{i}\right|<\mu$, and then apply the induction hypothesis to find a bound $g_{i}$ for each $F_{i}$ and then a bound $g$ for all the $g_{i}$. So we may assume that $|\mathfrak{a}|^{+}<\mu=\operatorname{cf}(\mu)<\lambda$.

If $F$ is enumerated as $\left\langle f_{\alpha}: \alpha<\mu\right\rangle$, then we may use the induction hypothesis to define inductively $f_{\alpha}^{*}$ which is a $<_{J}$-upper bound for the set $\left\{f_{\beta} \mid \beta<\alpha\right\} \cup\left\{f_{\beta}^{*} \mid \beta<\alpha\right\}$. Then $\left\langle f_{\alpha}^{*}: \alpha<\mu\right\rangle$ is $<J^{\text {-increasing, and a }}$ 
bound for $\left\{f_{\alpha}^{*} \mid \alpha<\mu\right\}$ will be a bound for $\left\{f_{\alpha} \mid \alpha<\mu\right\}$. Relabeling, we may as well assume that we are trying to find an upper bound for a sequence $\left\langle f_{\alpha}: \alpha<\mu\right\rangle$ which is $<_{J}$-increasing.

We will define a sequence of functions $g_{\beta}$ such that $\beta<\gamma \Rightarrow g_{\beta}<g_{\gamma}$, in such a way that for some $\beta<|\mathfrak{a}|^{+}$the function $g_{\beta}$ will be a bound for $\vec{f}$. Set $g_{0}(\theta)=0$ for all $\theta$, and for limit $\lambda<|\mathfrak{a}|^{+}$we define $g_{\lambda}=\sup _{\beta<\lambda} g_{\beta}(\theta)$; $g_{\lambda} \in \prod \mathfrak{a}$ because $\operatorname{cf}(\theta)=\theta>|\mathfrak{a}|^{+}$for all $\theta \in \mathfrak{a}$.

Suppose that we have defined $g_{\beta}$. If it fails to be a bound for the sequence $\vec{f}$ then $\left\{\theta \mid f_{\alpha(\beta)}(\theta)>g_{\beta}(\theta)\right\} \in J^{+}$for some $\alpha(\beta)<\mu$. By the definition of $J$ we may choose an ultrafilter $D$ such that $g_{\beta}<_{D} f_{\alpha(\beta)}$ and $\operatorname{cf}\left(\prod \mathfrak{a} / D\right) \geq \lambda$ (so necessarily $D \cap J=\emptyset !$ ) and we will then define $g_{\beta+1}$ to be some function such that $g_{\beta+1}>g_{\beta}$ and $g_{\beta+1}$ is an upper bound for $\vec{f}$ in $\prod \mathfrak{a} / D$. The key point here is that since $D \cap J=\emptyset$ and $\vec{f}$ is $<_{J}$-increasing we have $g_{\beta}<{ }_{D} f_{\alpha}<_{D} g_{\beta+1}$ for all $\alpha \geq \alpha(\beta)$, so that in particular there exists $\theta$ with $g_{\beta}(\theta)<f_{\alpha}(\theta)<g_{\beta+1}(\theta)$.

Suppose for a contradiction that the construction of $\vec{g}$ runs for $|\mathfrak{a}|^{+}$many steps. Choose $\alpha \geq \sup _{\beta<|\mathfrak{a}|^{+}} \alpha(\beta)$. Then for all $\beta<|\mathfrak{a}|^{+}$there exists $\theta \in \mathfrak{a}$ with $g_{\beta}(\theta)<f_{\alpha}(\theta)<g_{\beta+1}(\theta)$. Some $\theta$ must occur twice, but this is absurd because $\vec{g}$ is a sequence which increases on every coordinate.

Thus for some $\beta<|\mathfrak{a}|^{+}, g_{\beta}$ is a bound. This concludes the proof.

8.5. COROLlary. Let $\mathfrak{a}$ be a set of regular cardinals with $|\mathfrak{a}|^{+}<\min (\mathfrak{a})$. Then:

(1) For every cardinal $\lambda, \operatorname{cf}\left(\prod \mathfrak{a} / D\right)<\lambda$ iff $D \cap J_{<\lambda} \neq \emptyset$.

(2) $\operatorname{pcf}(\mathfrak{a})$ has a maximum element.

Proof. The right to left direction in the first claim is immediate from the definition of $J_{<\lambda}$, so suppose that $D \cap J_{<\lambda}=\emptyset$. In this case it follows immediately from the $\lambda$-directedness of $\left(\prod \mathfrak{a},<_{J_{<\lambda}}\right)$ that $\left(\prod \mathfrak{a},<_{D}\right)$ is also $\lambda$-directed, so that clearly $\operatorname{cf}\left(\prod \mathfrak{a} / D\right) \geq \lambda$.

For the second claim suppose that $\operatorname{pcf}(\mathfrak{a})$ has no largest element, and let $\mu$ be the supremum of $\operatorname{pcf}(\mathfrak{a})$. Then $\mathfrak{a} \notin J_{<\lambda}$ for each $\lambda<\mu$, because $\mathfrak{a} \in J_{<\lambda} \Rightarrow \sup \operatorname{pcf}(\mathfrak{a}) \leq \lambda$. So $\bigcup_{\lambda<\mu} J_{<\lambda}$ is a proper ideal, and we may choose an ultrafilter $D$ disjoint from it. But now $\operatorname{cf}\left(\prod \mathfrak{a} / D\right)=\lambda$ for some $\lambda<\mu$, and so $D \cap J_{<\lambda^{+}} \neq \emptyset$, contradicting the choice of $D$.

Now we turn our attention to problems about true cofinalities. We need a technical lemma about unbounded sequences modulo some ideal $I$; intuitively the lemma says that an unbounded sequence can be split into cofinal and bounded parts.

8.6. Lemma. Let $\mathfrak{a}$ be a set of regular cardinals, with $|\mathfrak{a}|^{+}<\min (\mathfrak{a})$. Let $I$ be an ideal on $\mathfrak{a}$, let $\mu=\operatorname{cf}(\mu)>|\mathfrak{a}|^{+}$, and $\left\langle f_{\alpha}: \alpha<\mu\right\rangle$ be increasing and 
unbounded in $\left(\prod \mathfrak{a},<_{I}\right)$. Then there is a sequence $\left\langle\mathfrak{b}_{\gamma}: \gamma<\mu\right\rangle$ of sets in $I^{+}$ such that:

(1) $\gamma<\delta \Rightarrow \mathfrak{b}_{\gamma} \subseteq_{I} \mathfrak{b}_{\delta}$

(2) $\left\langle f_{\alpha} \mid \mathfrak{b}_{\gamma}: \alpha<\mu\right\rangle$ is cofinal in $\prod \mathfrak{b}_{\gamma} / I$ for each $\gamma$.

(3) $\vec{f}$ is bounded modulo the ideal generated by $I$ and the $\mathfrak{b}_{\gamma}$ 's.

Proof. As in the proof of Lemma 8.4 we will build a sequence $\vec{g}$ of functions which are increasing on each coordinate, where $g_{0}=0$ and we take the pointwise supremum at each limit stage $\beta<|\mathfrak{a}|^{+}$. Suppose we have defined $g_{\beta}$. Then since $\vec{f}$ is unbounded, the set $\mathfrak{b}_{\gamma}^{\beta}=\left\{\theta \mid g_{\beta}(\theta)<f_{\gamma}(\theta)\right\}$ is in $I^{+}$for all $\gamma$ sufficiently large (say $\gamma \geq \gamma(\beta)$ ). Consider $\left\langle\mathfrak{b}_{\gamma}^{\beta}: \gamma \geq \gamma(\beta)\right\rangle$ as a candidate for the desired sequence $\overrightarrow{\mathfrak{b}}$; clearly it is positive and increasing modulo $I$, and what is more, $g_{\beta}$ will be a bound for $\vec{f}$ modulo the ideal generated by $I$ and $\left\langle\mathfrak{b}_{\gamma}^{\beta}: \gamma \geq \gamma(\beta)\right\rangle$.

So the construction is finished unless there is a $\gamma^{*}(\beta) \geq \gamma(\beta)$ such that $\left\langle f_{\alpha} \mid \mathfrak{b}_{\gamma^{*}(\beta)}^{\beta}: \alpha<\mu\right\rangle$ fails to be cofinal. In this case we will choose $g_{\beta+1}>g_{\beta}$ to be a witness to this failure of cofinalness, which is to say that $\left\{\theta \in \mathfrak{b}_{\gamma^{*}(\beta)}^{\beta} \mid g_{\beta+1}(\theta)>f_{\alpha}(\theta)\right\} \in I^{+}$for all $\alpha$. The key point is that (since the $\mathfrak{b}_{\gamma}^{\beta}$ are increasing modulo $I$ with $\gamma$ ) for all $\gamma \geq \gamma^{*}(\beta)$ and all $\alpha$ we have $\left\{\theta \in \mathfrak{b}_{\gamma}^{\beta} \mid g_{\beta+1}(\theta)>f_{\alpha}(\theta)\right\} \in I^{+}$.

Now suppose that the construction runs for $|\mathfrak{a}|^{+}$many steps. Choose $\gamma \geq \sup _{\beta<|\mathfrak{a}|^{+}} \gamma^{*}(\beta)$. Applying the conclusion of the last paragraph for $\alpha=\gamma$ gives $\left\{\theta \in \mathfrak{b}_{\gamma}^{\beta} \mid g_{\beta+1}(\theta)>f_{\gamma}(\theta)\right\} \in I^{+}$, so in particular there is $\theta \in \mathfrak{a}$ such that $g_{\beta}(\theta)<f_{\gamma}(\theta)<g_{\beta+1}(\theta)$. This leads to a contradiction exactly as in the proof of Lemma 8.4.

This shows that at some stage $\beta<|\mathfrak{a}|^{+}$the construction terminates, giving a sequence $\overrightarrow{\mathfrak{b}}$ as desired.

Using the facts above we can derive the key fact about pcf which is being used in this paper.

8.7. Theorem. Let $\theta=\max \operatorname{pcf}(\mathfrak{a})$. Then $\operatorname{tcf}\left(\prod \mathfrak{a} / J_{<\theta}\right)=\theta$.

Proof. Define $J=J_{<\theta} \cup\left\{\mathfrak{b} \notin J_{<\theta} \mid \operatorname{tcf}\left(\prod \mathfrak{b} / J_{<\theta}\right)=\theta\right\}$. It is easy to check that $J$ is a (possibly improper) ideal. If $\mathfrak{a} \in J$ we are done, otherwise choose an ultrafilter $D$ on a with $J \cap D=\emptyset$. Since $J_{<\theta} \cap D=\emptyset$ and $\theta=\max \operatorname{pcf}(\mathfrak{a})$ it is easy to see that $\operatorname{cf}\left(\prod \mathfrak{a} / D\right)=\theta$.

Choose $\left\langle f_{\alpha}: \alpha<\theta\right\rangle$ which is increasing and cofinal in $\left(\prod \mathfrak{a},<_{D}\right)$. Since $D \cap J_{<\theta}=\emptyset$ and $\prod \mathfrak{a} / J_{<\theta}$ is $\theta$-directed we may also assume that $\vec{f}$ is $<_{J_{<\theta^{-}}}$ increasing. It is easy to see that since $\vec{f}$ is cofinal modulo $D$ it is unbounded modulo $J_{<\theta}$, so that we may apply Lemma 8.6. 
Let $\overrightarrow{\mathfrak{b}}$ be the sequence of sets given by Lemma 8.6. If $\mathfrak{b}_{\gamma} \notin D$ for all $\gamma$ then the ideal generated by $J_{<\theta}$ and $\overrightarrow{\mathfrak{b}}$ is contained in the ideal dual to $D$, contradicting the choice of $\vec{f}$ to be cofinal modulo $D$. If on the other hand there is a $\gamma$ with $\mathfrak{b}_{\gamma} \in D$, then $\left\langle f_{\alpha} \mid \mathfrak{b}_{\gamma}: \alpha<\theta\right\rangle$ witnesses that $\operatorname{tcf}\left(\prod \mathfrak{b}_{\gamma} / I\right)=J_{\theta}$, so $\mathfrak{b}_{\gamma} \in D \cap J$, contradicting the choice of $D$ disjoint from $J$.

This contradiction shows that $\mathfrak{a} \in J$, hence $\operatorname{tcf}\left(\prod \mathfrak{a} / J_{<\theta}\right)=\theta$ and we are done.

\section{References}

[BuMa] M. Burke and M. Magidor, Shelah's pcf theory and its applications, Ann. Pure Appl. Logic 50 (1990) 207-254.

[Ca] T. Carlson, unpublished.

[DeJe] K. J. Devlin and R. B. Jensen, Marginalia to a theorem of Silver, in: Proc. ISILC Logic Conference (Kiel, 1974), Springer, Berlin, 1975, 115-142.

[Sh:b] S. Shelah, Proper Forcing, Springer, Berlin, 1982.

[Sh:g] —, Cardinal Arithmetic, Clarendon Press, Oxford, 1994.

[Sh410] -, More on cardinal arithmetic, Arch. Math. Logic 32 (1993) 399-428.

[Sh420] - Advances in cardinal arithmetic, in: Finite and Infinite Combinatorics in Sets and Logic, Kluwer, New York, 1993, 355-383.

[Sh598] —, More on covering lemma, in preparation.

[Sh:E12] -, Analytic guide, math.LO/9906022.

Institute of Mathematics

The Hebrew University of Jerusalem

91904 Jerusalem, Israel

E-mail: shelah@math.huji.ac.il
Department of Mathematics Rutgers University New Brunswick, NJ 08854, U.S.A. 